\title{
ID1 regulates U87 human cell proliferation and invasion
}

\author{
PIN GUO $^{1 *}$, JIN LAN $^{1 *}$, JIANWEI GE $^{1}$, QING MAO $^{1,2}$ and YONGMING QIU ${ }^{1,2}$ \\ ${ }^{1}$ Department of Neurosurgery, Ren Ji Hospital, Shanghai Jiao Tong University School of Medicine; \\ ${ }^{2}$ Shanghai Institute of Head Trauma, Shanghai 200127, P.R. China
}

Received January 25, 2013; Accepted July 8, 2013

DOI: $10.3892 / 01.2013 .1507$

\begin{abstract}
Despite therapeutic advances, the prognosis of patients diagnosed with malignant glioma has not improved in recent years. In particular, the molecular mechanisms that mediate glioma invasion remain poorly understood. The importance of ID1 in promoting tumor invasion and metastasis has recently emerged and a role for ID1 as a possible molecular marker of tumor aggressiveness has been proposed. To investigate the biological function of ID1 in glioblastomas, ID1-silenced U87 glioblastoma multiforme (GBM) cells were constructed using a small hairpin RNA (shRNA) sequence. The effect of the knockdown of ID1 on proliferation and invasion in these cells was analyzed using the 5-bromo-2'-deoxy-uridine cell proliferation, Transwell invasion, scratch and cell adhesion assays. Compared with the controls, the U87 cells expressing ID1-shRNA exhibited a significantly decreased proliferation and invasion capacity $(\mathrm{P}<0.05)$, as well as increased cell adhesion. Furthermore, silencing ID1 reduced the expression of c-Myc, cyclin D1 and $\beta$-catenin, while increasing E-cadherin expression in U87 cells. This study showed that ID1 regulates the metastatic potential of GBM cells by controlling the epithelial-mesenchymal transition. Therefore, ID1 is a potential prognostic indicator and therapeutic target in glioblastomas.
\end{abstract}

\section{Introduction}

Despite advances in glioma treatments, including neurosurgery, radiotherapy and chemotherapy, the prognosis of patients diagnosed with malignant glioma has not improved in recent years (1-4). The poor prognosis of glioblastoma multiforme (GBM) is mainly due to tumor cell invasion of the brain tissue

Correspondence to: Professor Yongming Qiu or Dr Qing Mao, Department of Neurosurgery, Ren Ji Hospital, Shanghai Jiao Tong University School of Medicine, 1630 Dongfang Road, Shanghai 200127, P.R. China

E-mail: qiuzhoub@hotmail.com

E-mail:maoq@netease.com

${ }^{*}$ Contributed equally

Key words: ID1, glioblastoma cell, invasion, proliferation beyond the resected areas (5-7). Moreover, brain tumors are resistant to current therapies (6).

Glioma invasion is a complex cellular phenomenon that involves changes in the intracellular and extracellular biomechanical systems (4). Although our understanding of glioma oncogenesis is steadily improving, the molecular mechanisms that mediate glioma invasion remain poorly understood (8). There have been numerous studies describing the extracellular factors involved in glioma cell invasion, including $\beta$-catenin, c-Myc and cyclin D1 (9-11). However, the intracellular and molecular mechanisms that mediate glioma invasion require further elucidation in order to identify new drug targets.

ID proteins (inhibitors of DNA binding/differentiation) are helix-loop-helix transcription factors $(12,13)$. The reactivation of the ID proteins, particularly ID1, promotes the development of several tumor types, including high-grade astrocytoma, prostate and breast cancers and non-small cell lung carcinoma (14-17). ID1 controls the expression of a large number of genes that mediate important cellular processes by inhibiting the activity of bHLH proteins (18-20). The main role of ID1 is to inhibit cell differentiation (18). In addition, loss of differentiation, unrestricted proliferation and increased cell invasion are hallmarks of malignancy. By maintaining an immature phenotype, ID1 enhances cell proliferation and invasion. Therefore, ID1 overexpression may induce invasion in several cancer types (21-23). The importance of ID1 in promoting tumor invasion and metastasis has emerged and a role as a possible molecular marker of tumor aggressiveness has been proposed (22). However, the role of ID1 in GBM is poorly understood.

In the present study, ID1-small hairpin RNA (shRNA)-expressing U87 cells and controls were constructed, and Transwell invasion and scratch assays were performed to analyze the effect of the knockdown of ID1 on cell invasion.

\section{Materials and methods}

Cell culture. The U87 human glioma cell line was obtained from the American Type Culture Collection (Manassas, VA, USA). The cells were grown in Dulbecco's modified Eagle's medium (DMEM; Gibco BRL, Gaithersburg, MD, USA) supplemented with $10 \%$ fetal bovine serum (FBS) at $37^{\circ} \mathrm{C}$ in a humidified atmosphere containing $5 \% \mathrm{CO}_{2}$.

$q P C R$. The U87 cells were washed three times with ice-cold phosphate-buffered saline (PBS) and total RNA was extracted 
using TRIzol (Invitrogen, Carlsbad, CA, USA). An equal amount of total RNA was used for first-strand cDNA synthesis using the oligo-dT primer and M-myeloblastosis virus reverse transcriptase XL (Promega, Madison, WI, USA) in a reaction volume of $25 \mu \mathrm{l}$, according to the manufacturer's instructions. Synthesized first-strand cDNA $(2 \mu \mathrm{l})$ was used for each PCR reaction.

qPCR experiments were performed using the SYBR Green PCR Master Mix (Applied Biosystems, Foster City, CA, USA). The PCR products were subjected to melting curve analysis to exclude the synthesis of non-specific products. The $\mathrm{Ct}$ value was quantified using a standard curve for the specific gene and relatively quantified using GAPDH as an internal reference control. The $\mathrm{Ct}$ value was then normalized to the average expression levels of undifferentiated samples, calculated according to the $2^{-\Delta \Delta \mathrm{Ct}}$ method. All experiments were performed in triplicate.

Western blotting. Cellular proteins $(30 \mu \mathrm{g})$ were subjected to $12 \%$ SDS polyacrylamide gel electrophoresis (SDS-PAGE) and transferred onto Hybond ECL nitrocellulose membranes (Amersham, Piscataway, NJ, USA). Subsequent to being washed with $0.1 \%$ TBS-T, the membranes were blocked in $5 \%$ skimmed milk in TBS-T for $1 \mathrm{~h}$ at room temperature, then incubated with the appropriate antibody [1/500 dilution, ID1 antibody sc-488 (Santa Cruz Biotechnology, Inc., Santa Cruz, CA, USA); 1/1,000 dilution $\beta$-actin antibody (Abcam, London, England); 1/1,000 dilution, cyclin D1 antibody (Cell Signaling Technology, Danvers, MA, USA); 1/1,000 dilution, c-Myc antibody (Cell Signaling Technology); 1/1,000 dilution, $\beta$-catenin antibody (Abcam); 1/500 dilution, E-cadherin antibody (Cell Signaling Technology)] diluted in the same buffer overnight at $4^{\circ} \mathrm{C}$. Subsequent to being washed with $0.1 \%$ TBS-T, the membranes were incubated with horseradish peroxidase-conjugated anti-rabbit or anti-mouse antibody, as appropriate (1/10,000 dilution; Sigma, St. Louis, MO, USA), for $2 \mathrm{~h}$ at room temperature. After washing with $0.1 \%$ TBS-T, specific protein bands were detected using western blotting detection reagents (Odyssey; Licor, Lincoln, NE, USA).

Construction of U87 cells. The pGIPZ expression vector (Thermo Scientific, Waltham, MA, USA) carrying the ID1-shRNA coding sequence (shRNA sequence, TCGGAATCCGAAGTTGGAA) and the control empty vector were transfected into the U87 cells using Lipofectamine 2000 (Invitrogen), according to the manufacturer's instructions. Two days after transfection, $800 \mu \mathrm{g} / \mathrm{ml}$ puromycin (Sigma) was added to the growth medium for the selection of stable ID1-shRNA-expressing cells. Colonies expressing marked green fluorescence were selected for further studies.

BrdU proliferation of cells. Cell proliferation was assessed by 5-bromo-2'-deoxy-uridine (BrdU) incorporation using a Cell Proliferation ELISA, BrdU kit (Roche Applied Science, Indianapolis, IN, USA) according to the manufacturer's instructions. The cells were seeded onto a 96-well plate at a density of $1 \times 10^{5}$ cells/well in $100 \mu \mathrm{l}$ culture medium and incubated at $37^{\circ} \mathrm{C}$ for $6,12,24$ or $48 \mathrm{~h}$. BrdU labeling solution was then added to a final concentration of $10 \mu \mathrm{M}$ and the cells were incubated for an additional $2-4 \mathrm{~h}$ at $37^{\circ} \mathrm{C}$. The medium was then removed and FixDenat (200 $\mu \mathrm{l} /$ well) was added to the cells and incubated for $30 \mathrm{~min}$ at $25^{\circ} \mathrm{C}$. The FixDenat solution was then completely removed and $100 \mu \mathrm{l} /$ well anti-BrdU-POD working solution was added and incubated for $90 \mathrm{~min}$ at $25^{\circ} \mathrm{C}$ The antibody conjugate was then removed by flicking and the wells were washed three times with $200 \mu \mathrm{l} /$ well washing solution. Substrate solution (100 $\mu \mathrm{l} /$ well) was added and the cells were incubated at $25^{\circ} \mathrm{C}$ until color development was sufficient for photometric detection (after 6, 12, 24 and $48 \mathrm{~h}$ ). The absorbance [optical density (OD)] at $450 \mathrm{~nm}$ was measured using a microplate reader.

Cell adhesion assay. A 96-well plate was coated with Matrigel and incubated at $37^{\circ} \mathrm{C}$ for $1 \mathrm{~h}$. The U87 cells were then plated at $5 \times 10^{4}$ cells/well in serum-free MEM and the plate was incubated for $30 \mathrm{~min}$ at $37^{\circ} \mathrm{C}$, followed by a gentle rinse with PBS to remove non-adherent cells. The cells were then fixed for $20 \mathrm{~min}$ with $3.5 \%$ formalin, stained with $0.5 \%$ crystal violet for $1 \mathrm{~h}$ and rinsed twice with PBS. The absorbance of the test samples and blank controls was measured at $594 \mathrm{~nm}$ using a microplate reader. The OD value of each test sample was designated the measured value and that of the blank was designated the blank value. The final value $=$ measured value - blank value.

Transwell invasion assay. Matrigel (25 mg reconstituted basement membrane) was added onto a polyvinylpyrrolidone-free polycarbonate filter (Nuclepore; Whatman, Maidstone, UK) and dried. The cells were harvested following brief exposure to $1 \mathrm{mM}$ EDTA, then washed with DMEM containing $0.1 \%$ bovine serum albumin and added to Boyden chambers ( $2 \times 10^{5}$ cells/chamber). The chambers were incubated for $24 \mathrm{~h}$ at $37^{\circ} \mathrm{C}$. The cells that traversed the Matrigel layer and became attached to the filter were stained using a Diff-Quik kit (Dade Diagnostics, Aguada, PR, USA) and five randomized fields were counted. The mean $\pm \mathrm{SE}$ was calculated for three independent experiments.

Scratch assay. The cells were cultured to $90 \%$ confluency in six-well plates, then a thin scratch (wound) was made in the central area using a $10-\mathrm{ml}$ pipette tip. Detached and damaged cells were carefully removed with PBS and the medium was replaced with serum-free medium. Wound closure was observed by light microscopy and images were captured at the indicated time points.

Immunofluorescence. The cells were cultured on glass coverslips in 35-mm diameter dishes, washed with PBS and fixed with $4 \%$ p-formaldehyde for $15 \mathrm{~min}$. Subsequent to being washed with PBS, the cells were incubated with $0.1 \%$ Triton X-100 for 25 min, washed three times with PBS and blocked with $10 \%$ goat serum for $30 \mathrm{~min}$ at room temperature. The cells were then incubated with an anti-actin antibody $(1 / 100$; Abcam) in a humidified chamber either overnight at $4^{\circ} \mathrm{C}$ or for $2 \mathrm{~h}$ at room temperature, followed by incubation with Alexa 488-conjugated goat anti-mouse antibody (1/200; Molecular Probes, Eugene, OR, USA) for $1 \mathrm{~h}$ at room temperature in a humidified chamber. DAPI staining was performed to identify the cell nuclei, and the cells were observed using a confocal Lasersharp 2000 version 5.1 microscope (Carl Zeiss, Jena, 


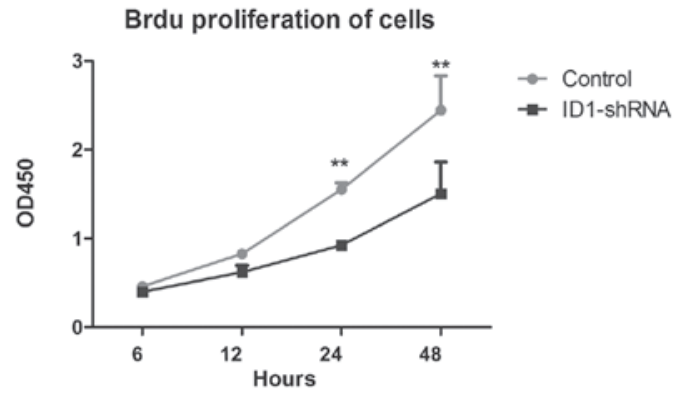

Figure 1. ID1-knockdown inhibits U87 cell proliferation. The numbers of ID1-shRNA-expressing and control U87 cells were compared at 6, 12, 24 and $48 \mathrm{~h}$ post-incubation with BrdU. At 24 and $48 \mathrm{~h}$, the control group cells had higher OD values ${ }^{* *} \mathrm{P}<0.01$, vs. ID1-shRNA). shRNA, small hairpin RNA; OD, optical density.

Germany) equipped with a Plan-Apochromat 63x/1.40 oil objective lens. Confocal images were acquired using LSM 5102.3 software (Alexa 488 emission was at $519 \mathrm{~nm}$ and Alexa 568 emission was at $603 \mathrm{~nm}$ ).

Statistical analysis. Data are represented as the mean $\pm \mathrm{SE}$ from at least three independent experiments. All data were analyzed using an independent samples t-test using GraphPad Prism 5 software (San Diego, CA, USA). P<0.05 were considered to indicate a statistically significant difference.

\section{Results}

ID1-knockdown inhibits the proliferation of U87 cells. Our previous study showed that ID1 is highly expressed in several GBM cell lines, including A172, T98g, U251 and U87; of these, the U87 cells have the highest level of ID1 expression. Therefore, ID1 was knocked down in the U87 cells using ID1-shRNA expression and the changes in cell proliferation and invasion were measured between the ID1-silenced and control cells transfected with empty vector. It appears that ID1 expression was reduced in the U87 cells following shRNA-ID1 transfection (24).

Cell proliferation is necessary for the development of all types of cancer, including gliomas. A previous study demonstrated that ID1 promotes tumor progression, although there is no direct evidence that ID1 is involved in GBM cell proliferation (25). The BrdU cell proliferation assay was therefore used to analyze the role of ID1 in GBM cell proliferation. As shown in Fig. 1, there were significant differences in cell proliferation between the ID1-silenced and control cells at the 24- and 48-h time points; decreased ID1 expression correlated with a reduction in U87 cell proliferation. These data suggest that ID1 has a role in blocking aberrant U87 cell proliferation.

ID1-knockdown reduces the invasiveness of U87 cells. Transwell invasion and scratch assays were used to measure the rate of invasion of the ID1-silenced and control U87 cells. The Transwell invasion assay showed that fewer ID1-shRNA-expressing U87 cells passed through a polycarbonate membrane compared with the controls $(\mathrm{P}<0.0001$; Fig. 2A and B). The scratch assay showed that the there was no significant difference in wound healing between the two groups of cells after $6 \mathrm{~h}$. However, after $36 \mathrm{~h}$, there was almost complete wound healing in the control cells, but not in the U87 cells (Fig. 2C). This result is consistent with the result of the Transwell invasion assay, i.e., that wound healing and migration are inhibited by reduced ID1 expression. These data suggest that ID1-knockdown blocks U87 cell invasion.

ID1-knockdown enhances U87 cell adhesion. Reduced adhesion is necessary for the increase in cell mobility and invasion capacity. A cell adhesion assay was used to compare the adhesive properties of ID1-silenced and control cells. As shown in Fig. 2D, the U87 cells with ID1-knockdown were more adhesive compared with the control cells, i.e., a change in ID1 expression led to a change in cell adhesion. Compared with the controls, the ID1-silenced U87 cells showed more marked adhesive properties. This is consistent with higher levels of cell invasion in normal U87 cells.

Effects of ID1-knockdown on U87 morphology and cytoskeleton. In order to observe the morphological and cytoskeletal changes in the U87 cells following the silencing of ID1, actin filaments were observed by immunofluorescence and confocal scanning laser microscopy in the treated and control cells. In the control cells, actin was localized and highly expressed on pseudopodia around the nucleus and on stress fibers. However, the U87 cells expressing ID1-shRNA became flatter and smaller, with shorter lamellipodia compared with the controls (Fig. 3). Thus, cytoskeletal changes due to ID1 downregulation are associated with altered tumor cell invasion properties. These data support the hypothesis that U87 cell invasion is reduced following the knockdown of ID1.

IDI regulates expression of $\beta$-catenin, cyclin D1, $c$-Myc and $E$-cadherin. Several signaling molecules have been reported to contribute to the increased invasion and proliferation properties of certain types of cancer; these include c-Myc, cyclin D1 and $\beta$-catenin. For example, the $\beta$-catenin pathway is critical in glioma tumor invasion (10), while c-Myc and cyclin D1 are also involved in several pathways that promote GBM invasion. Therefore, the expression levels of these proteins were measured in the ID1-silenced and control cells in order to determine whether protein levels correlate with invasiveness. In the U87 cells, ID1-knockdown led to the reduced expression of c-Myc, cyclin D1 and $\beta$-catenin (Fig. 4). The epithelial-mesenchymal transition (EMT) increases the invasive capacity of tumor cells (26). As previously reported, the level of E-cadherin expression is a critical marker of EMT (27). The present data showed that E-cadherin expression was increased following ID1-knockdown, i.e., a reduction in ID1 expression inhibits EMT and thus invasiveness in U87 cells.

\section{Discussion}

GBM is the most common type of central nervous system malignancy and the prognosis of patients with GBM is not improved by standard treatments $(1,3)$. The majority of GBMs are poorly differentiated; this is linked to tumor aggression and lethality (28). Malignant glioma cell proliferation and invasion are key stages in cancer progression that affect patient mortality (29). Clinically, there are limited therapeutic 
A

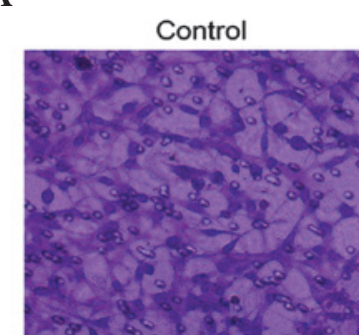

C

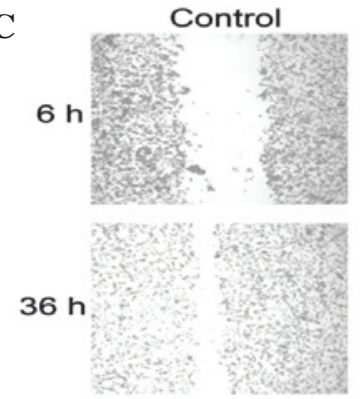

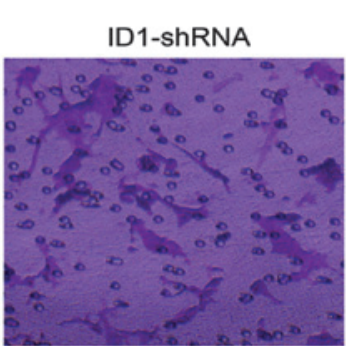

ID1-shRNA

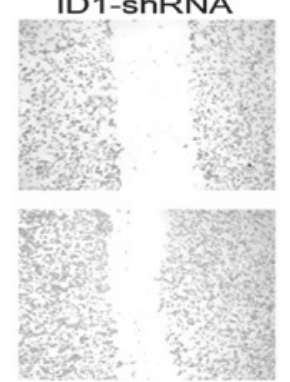

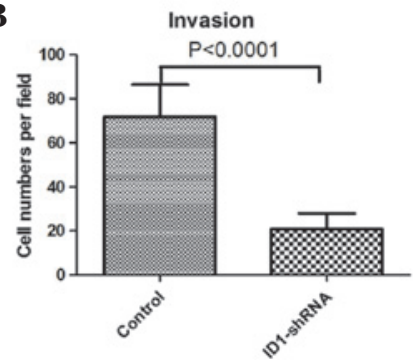

D

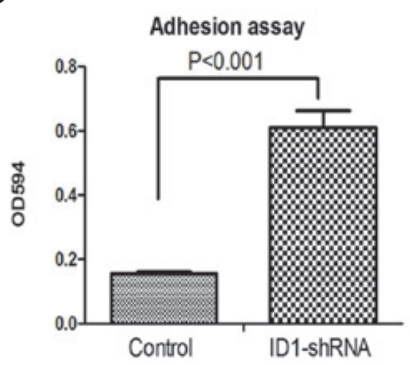

Figure 2. ID1-knockdown inhibits U87 cell invasion. (A) Cells were stained with crystal violet and then observed by light microscopy (magnification, x100). (A and B) Transwell invasion assay showing differences in invasiveness between ID1-shRNA-expressing and control U87 cells. (C) Scratch assay comparing the wound healing properties of ID1-shRNA-expressing and control U87 cells (magnification, x20). (D) Adhesion assay comparing the adhesion ability of ID1-shRNA-expressing and control U87 cells.

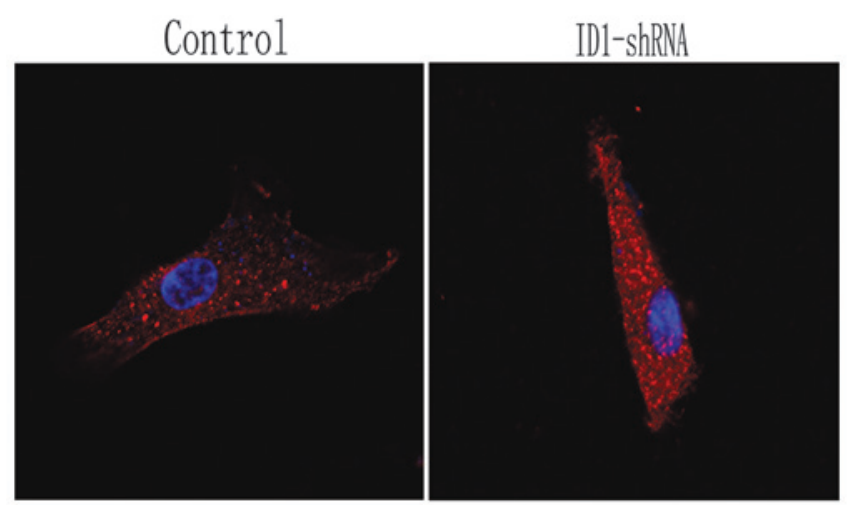

Figure 3. ID1-knockdown induces alterations in the actin cytoskeleton of U87 cells. ID1-shRNA-expressing and control U87 cells were stained with an anti-actin antibody and observed by confocal microscopy (magnification, $\mathrm{x} 400)$.

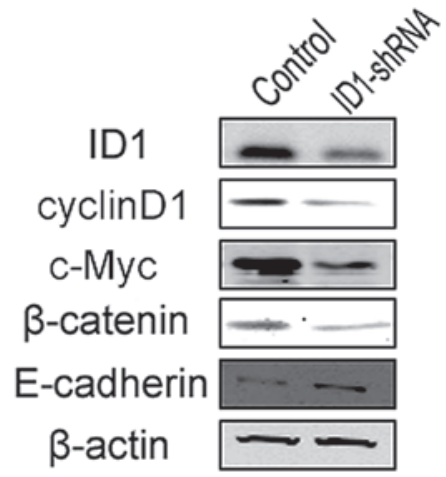

Figure 4. ID1 regulates factors that are involved in the proliferation and invasion of U87 cells. The expression levels of CyclinD1, C-myc and $\beta$-catenin were reduced in ID1-shRNA-expressing U87 cells, while E-cadherin expression was increased compared with the controls. interventions for malignant glioma. Therefore, more research into the mechanisms of GBM invasion is essential for the development of a curative therapy.

ID1, an inhibitor of basic helix-loop-helix transcription factors, has been shown to be a key regulator of a number of steps in cancer progression $(12,13)$. Moreover, ID1 inhibits cell differentiation and promotes invasion in several types of malignant cancers, including breast and prostate cancers and non-small cell lung carcinoma $(14,16,23)$. Generally, ID1 contributes to tumorigenesis by inhibiting cell differentiation, stimulating proliferation, enhancing invasion and facilitating tumor neoangiogenesis $(30,31)$. Perk et al, however, suggested that ID1 function may depend on cell type (13). Meng et al demonstrated that ID1 induces differentiation in mouse embryonic carcinoma P19CL6 cells (32). Furthermore, Geng et al reported that ID1 enhances docetaxel cytotoxicity in prostate cancer cells through p21 inhibition and suggested that ID1 is a novel prognostic marker and therapeutic target in prostate cancer chemotherapy (33). There have been several studies of ID1 in glioma. Vandeputte et al reported that ID expression is lower in low-grade astrocytoma compared to high-grade astrocytoma and therefore inferred that ID1 expression levels are associated with the grade of glioma (17). Anido et al identified a cell population enriched with glioma-initiating cells (GICs) that express high levels of ID1 and suggested that high ID1 levels are associated with a poor prognosis in GBM patients (34). By contrast, Barrett et al reported that an improved prognosis is associated with higher ID1 expression in a preneural subgroup of GBM, even though ID1 overexpression correlates with increased self-renewal in GICs (35). These contradictory studies show that further investigations are required to determine the function of ID1 in GBM.

Since ID1 promotes cell proliferation and invasion, it has been proposed as an attractive target for cancer therapy. 
Therefore, in the present study, an ID1-shRNA transfection system was established for U87 cells to investigate the correlation between ID1 expression and biological outcome. Using Transwell invasion and scratch assays, it was observed that ID1-shRNA-expressing U87 cells have a poorer invasion ability. In addition, it was demonstrated that compared with ID1-silenced U87 cells, the controls had poorer adhesion properties. The effect of ID1 silencing on cell proliferation was also investigated, as this contributes to tumor invasion. The BrdU proliferation assay was used to analyze cell proliferation in the U87 cells and ID1-knockdown was observed to lead to decreased proliferation.

In general, cell movement requires morphological changes that involve breaking down and reforming cytoskeletal filaments (36). Therefore, to determine whether changes in ID1-mediated cell invasion correlate with cytoskeletal alterations, actin filaments were visualized by immunofluorescence and changes in cell shape were determined following ID1-knockdown. The ID1-silenced cells had fewer lamellipodia and became rounder and smaller compared with the control cells. Such changes in cell morphology are associated with increased invasiveness and may be a consequence of the dysregulation of ID1 signaling components mediating proliferation and invasion (36). Factors such as cyclin D1, c-Myc and $\beta$-catenin have been shown to promote GBM cell invasion. Therefore, the expression of these proteins was examined in the ID1-silenced and control U87 cells (37). These proteins were all observed to be downregulated in the ID1-shRNA-expressing U87 cells. By contrast, ID1-knockdown increased E-cadherin protein levels, which are considered to be a marker of EMT. ID1 silencing may inhibit the process of EMT, which is pivotal in cell invasion. The present results therefore suggest that ID1-knockdown may inhibit U87 glioma cell proliferation and invasion.

The present study demonstrated that the loss or inhibition of ID1 expression may be important in GBM cell proliferation and invasion. However, the specific mechanism through which ID1 regulates these GBM cell properties requires further research, which may lead to the identification of new strategies and therapeutic targets for glioma treatment.

\section{Acknowledgements}

This study was supported by a project of building hospital clinical key disciplines (no. RJ.4101307) and a grant from the Shanghai government (no. 0952nm03900).

\section{References}

1. Ferguson SD: Malignant gliomas: diagnosis and treatment. Dis Mon 57: 558-569, 2011.

2. Onishi M, Ichikawa T, Kurozumi K and Date I: Angiogenesis and invasion in glioma. Brain Tumor Pathol 28: 13-24, 2011.

3. Rampling R, James A and Papanastassiou V: The present and future management of malignant brain tumours: surgery, radiotherapy, chemotherapy. J Neurol Neurosurg Psychiatry 75 (Suppl 2): ii24-ii30, 2004.

4. Salhia B, Tran NL, Symons M, Winkles JA, Rutka JT and Berens ME: Molecular pathways triggering glioma cell invasion. Expert Rev Mol Diagn 6: 613-626, 2006.

5. Harpold HL, Alvord EC Jr and Swanson KR: The evolution of mathematical modeling of glioma proliferation and invasion. J Neuropathol Exp Neurol 66: 1-9, 2007.
6. Okita Y, Narita Y, Miyakita Y, et al: Pathological findings and prognostic factors in recurrent glioblastomas. Brain Tumor Pathol 29: 192-200, 2012.

7. Park SG, Jung S, Ryu HH, et al: Role of 14-3-3-beta in the migration and invasion in human malignant glioma cell line U87MG. Neurol Res 34: 893-900, 2012.

8. de Groot JF and Gilbert MR: New molecular targets in malignant gliomas. Curr Opin Neurol 20: 712-718, 2007.

9. Bredel M, Bredel C, Juric D, et al: Functional network analysis reveals extended gliomagenesis pathway maps and three novel MYC-interacting genes in human gliomas. Cancer Res 65: 8679-8689, 2005.

10. Nager M, Bhardwaj D, Cantí C, Medina L, Nogués P and Herreros J: $\beta$-Catenin signalling in glioblastoma multiforme and glioma-initiating cells. Chemother Res Pract 2012: 192362, 2012.

11. Yue X, Lan F, Yang W, et al: Interruption of $\beta$-catenin suppresses the EGFR pathway by blocking multiple oncogenic targets in human glioma cells. Brain Res 1366: 27-37, 2010.

12. Sikder HA, Devlin MK, Dunlap S, Ryu B and Alani RM: Id proteins in cell growth and tumorigenesis. Cancer Cell 3: 525-530, 2003.

13. Perk J, Iavarone A and Benezra R: Id family of helix-loop-helix proteins in cancer. Nat Rev Cancer 5: 603-614, 2005.

14. Coppe JP, Itahana Y, Moore DH, Bennington JL and Desprez PY: Id-1 and Id-2 proteins as molecular markers for human prostate cancer progression. Clin Cancer Res 10: 2044-2051, 2004.

15. Gupta GP, Perk J, Acharyya S, et al: ID genes mediate tumor reinitiation during breast cancer lung metastasis. Proc Natl Acad Sci USA 104: 19506-19511, 2007.

16. Pillai S, Rizwani W, Li X, et al: ID1 facilitates the growth and metastasis of non-small cell lung cancer in response to nicotinic acetylcholine receptor and epidermal growth factor receptor signaling. Mol Cell Biol 31: 3052-3067, 2011.

17. Vandeputte DA, Troost D, Leenstra S, et al: Expression and distribution of id helix-loop-helix proteins in human astrocytic tumors. Glia 38: 329-338, 2002.

18. Benezra R, Davis RL, Lockshon D, Turner DL and Weintraub H: The protein Id: a negative regulator of helix-loop-helix DNA binding proteins. Cell 61: 49-59, 1990.

19. Kiewitz SD, Kruppa M, Riechers A, König B and Cabrele C: Recognition of the helix-loop-helix domain of the Id proteins by an artificial luminescent metal complex receptor. J Mol Recognit 21: 79-88, 2008.

20. Langlands K, Yin X, Anand G and Prochownik EV: Differential interactions of Id proteins with basic-helix-loop-helix transcription factors. J Biol Chem 272: 19785-19793, 1997.

21. Xie SQ, Zhang YH, Li Q, et al: 3-Nitro-naphthalimide and nitrogen mustard conjugate NNM-25 induces hepatocellular carcinoma apoptosis via PARP-1/p53 pathway. Apoptosis 17: 725-734, 2012.

22. Ciarrocchi A, Piana S, Valcavi R, Gardini G and Casali B: Inhibitor of DNA binding-1 induces mesenchymal features and promotes invasiveness in thyroid tumour cells. Eur J Cancer 47: 934-945, 2011.

23. Lin CQ, Singh J, Murata K, et al: A role for Id-1 in the aggressive phenotype and steroid hormone response of human breast cancer cells. Cancer Res 60: 1332-1340, 2000.

24. Guo Q, Guo P, Mao Q, et al: ID1 affects the efficacy of radiotherapy in glioblastoma through inhibition of DNA repair pathways. Med Oncol 30: 325, 2013.

25. Ling MT, Wang XH, Zhang XM and Wong YC: The multiple roles of Id-1 in cancer progression. Differentiation 74: 481-487, 2006.

26. Ahmad A, Aboukameel A, Kong D, et al: Phosphoglucose isomerase/autocrine motility factor mediates epithelial-mesenchymal transition regulated by miR-200 in breast cancer cells. Cancer Res 71: 3400-3409, 2011.

27. Wells A, Yates C and Shepard CR: E-cadherin as an indicator of mesenchymal to epithelial reverting transitions during the metastatic seeding of disseminated carcinomas. Clin Exp Metastasis 25: 621-628, 2008.

28. Borkar SA, Lakshmiprasad G, Subbarao KC, et al: Giant cell glioblastoma in the pediatric age group: Report of two cases. J Pediatr Neurosci 8: 38-42, 2013.

29. Qi S, Song Y, Peng Y, et al: ZEB2 mediates multiple pathways regulating cell proliferation, migration, invasion, and apoptosis in glioma. PLoS One 7: e38842, 2012.

30. Cheng YJ, Tsai JW, Hsieh KC, et al: Id1 promotes lung cancer cell proliferation and tumor growth through Akt-related pathway. Cancer Lett 307: 191-199, 2011. 
31. Tobin NP, Sims AH, Lundgren KL, Lehn S and Landberg G: Cyclin D1, Id1 and EMT in breast cancer. BMC Cancer 11: 417, 2011.

32. Meng Q, Jia Z, Wang W, Li B, Ma K and Zhou C: Inhibitor of DNA binding 1 (Id1) induces differentiation and proliferation of mouse embryonic carcinoma P19CL6 cells. Biochem Biophys Res Commun 412: 253-259, 2011.

33. Geng H, Rademacher BL, Pittsenbarger J, et al: ID1 enhances docetaxel cytotoxicity in prostate cancer cells through inhibition of p21. Cancer Res 70: 3239-3248, 2010.

34. Anido J, Sáez-Borderías A and Gonzàlez-Juncà A, et al: TGF- $\beta$ Receptor Inhibitors Target the CD44(high)/Id1(high) Glioma-Initiating Cell Population in Human Glioblastoma. Cancer Cell 18: 655-668, 2010.
35. Barrett LE, Granot Z, Coker C, et al: Self-renewal does not predict tumor growth potential in mouse models of high-grade glioma. Cancer Cell 21: 11-24, 2012.

36. Sarmiere PD and Bamburg JR: Regulation of the neuronal actin cytoskeleton by ADF/cofilin. J Neurobiol 58: 103-117, 2004.

37. Nager M, Bhardwaj D, Canti C, et al: $\beta$-catenin signaling in glioblastoma multiforme and glioma-initiating cells. Chemother Res Pract 2012: 192362, 2012. 